\title{
Journal of Biotechnology
}

Erratum

Erratum to "Effects of glucose and inoculum concentrations on production of bioactive molecules by Paenibacillus polymyxa RNC-D: A statistical experimental design" [J. Biotechnol. 150S (2010) S524]

N.F.G. Serrano ${ }^{1,2, *}$, S.I. Mussatto ${ }^{2}$, L.R. Rodrigues ${ }^{2}$, J.A. Teixeira ${ }^{2}$, C.O. Hokka ${ }^{1}$, C.P. Sousa ${ }^{1}$

${ }^{1}$ Federal University of Sao Carlos, Brazil

${ }^{2}$ University of Minho, Portugal

The publisher regrets that the Author name "S.I. Mussato" should be corrected as "S.I. Mussatto".

DOI of original article: http://dx.doi.org/10.1016/j.jbiotec.2010.09.841.

* Corresponding author. 